\title{
Fonder et acheter, étudier et s'approprier, construire et reconfigurer
}

Les trois temps de la transformation du « domaine copte » en église Saint-Alexandre Nevsky à Jérusalem (1856-1896)

\section{Elena Astafieva}

\section{(2) OpenEdition}

Journals

Édition électronique

URL : http://journals.openedition.org/ejts/5353

DOI : $10.4000 /$ ejts.5353

ISSN : $1773-0546$

Éditeur

EJTS

Référence électronique

Elena Astafieva, «Fonder et acheter, étudier et s'approprier, construire et reconfigurer », European

Journal of Turkish Studies [En ligne], 22 | 2016, mis en ligne le 01 août 2016, consulté le 16 février 2020.

URL : http://journals.openedition.org/ejts/5353 ; DOI : 10.4000/ejts.5353

Ce document a été généré automatiquement le 16 février 2020.

(c) Some rights reserved / Creative Commons license 


\section{Fonder et acheter, étudier et s'approprier, construire et reconfigurer}

Les trois temps de la transformation du « domaine copte » en église Saint-Alexandre Nevsky à Jérusalem (1856-1896)

\section{Elena Astafieva}

1 Cette recherche ${ }^{1}$ 'inscrit dans le cadre des études consacrées à la politique russe sur les territoires arabes de l'Empire ottoman et développées depuis la chute de l'URSS et l'ouverture de nombreuses archives russes. À partir des années 1960 en effet, les historiens anglo-saxons ont commencé à travailler sur la présence russe au Levant (Stavrou 1963 ; Hopwood 1969). Toutefois, à l'instar des historiens soviétiques ils n'ont pas eu alors accès à l'ensemble des sources russes impériales provenant soit de l'État, soit de l'Église orthodoxe ou d'autres religions, qui leur auraient permis d'analyser tous les aspects politico-religieux, diplomatiques et culturels de cette présence. Après 1991, la production historique russe ${ }^{2}$ se caractérise par une excellente connaissance des sources diplomatiques russes d'une part, mais d'autre part, elle ne cherche pas à inscrire la politique impériale au Levant dans un contexte international plus large (Lissovoj2006). Cependant, à partir des années 2010, de nouveaux travaux se fondent non seulement sur des sources issues des archives russes, mais aussi sur les archives diplomatiques européennes qui apportent une vision plus large des activités russes en Palestine et Syrie au cours du long XIX ${ }^{\mathrm{e}}$ siècle (Fairey 2012 ; Vovchenko 2013 ; Iakuchev 2013).

2 En effet, la Russie marque sa présence en Palestine dès 1847, avec la création de la Mission ecclésiastique de Jérusalem. Cette fondation s'inscrit dans un processus d'installation massive dans les provinces arabes de l'Empire ottoman. Ce qu'Henry Laurens désigne sous le terme de l'«invention de la Terre sainte» (Laurens 1999) intervient après la guerre syro-égyptienne de 1839 et le premier projet d'internationalisation de Jérusalem sous l'égide des principales puissances européennes (Prusse, Angleterre, France et Autriche). Toutefois, c'est seulement après la défaite de 
la guerre de Crimée (1854-1856) que la Russie conçoit et met en œuvre sa «politique orientale ». Cette politique devient beaucoup plus offensive qu'à l'époque précédente, car le but majeur de l'Empire russe est alors de retrouver son statut de grande puissance.

3 L'objectif de cet article, fondé sur l'étude de sources archivistiques collectées à Moscou et à Saint-Pétersbourg pendant l'été $2015^{3}$ est d'essayer de répondre aux questions suivantes : quelles sont les stratégies d'action de la Russie impériale dans la région proche-orientale dans la seconde moitié du XIX ${ }^{\mathrm{e}}$ siècle? Quels sont les acteurs institutionnels ou non-institutionnels qui mettent en œuvre ces stratégies dans la région? Comment leurs actions modifient-elles à la fois les rapports de force entre les acteurs au sein de l'espace impérial ottoman et les relations entre ces institutions en Russie?

4 Pour ce faire, je propose d'examiner les activités de ces acteurs menées à Jérusalem après la guerre de Crimée, en présentant le cas particulier de la transformation par les Russes d'un espace - "domaine copte » - situé au cœur de Jérusalem, près du SaintSépulcre, et en montrant que cette transformation s'opère en trois temps en lien avec trois types d'action :

- le temps de la fondation des institutions russes « en Orient » et de l'acquisition des terres à Jérusalem (1857-1864);

- le temps des fouilles archéologiques et de l'appropriation par la Russie des principaux symboles du christianisme (1882-1884);

- et enfin, le temps de la construction de l'église orthodoxe russe Saint-Alexandre Nevsky près du Saint-Sépulcre et de la reconfiguration de l'orthodoxie proche-orientale en marche (1885-1896...).

\section{Temps de fondation et d'acquisition}

$5 \quad$ Le $1^{\text {er }}$ mars 1857 , le prince Gortchakov, vice-chancelier d'État et ministre des Affaires étrangères russe, rédige un "projet d'instruction au chef de la Mission hiérosolymitaine $»^{4}$. Dans ce document Gortchakov, pour qui la "question d'Orient », après la défaite russe lors de la guerre de Crimée, était «l'idée dominante, sinon unique ", propose une série d'actions à mener "en Orient " [na Vostoke] après la signature du Traité de Paris le 18/30 mars 1856. Son but est de renforcer sur la scène internationale la position militaire et politique de la Russie, affaiblie par la neutralisation de la mer Noire et par l'interdiction à l'Empire russe d'avoir dans cette mer des pavillons de guerre et de construire au sud de ses frontières des bases militaires 5 .

6 Au début de son instruction, Gortchakov reconnaît que, «à l'heure actuelle », la Russie ne peut pas "reposer la question du Saint-Sépulcre", comme elle ne peut pas "protéger ouvertement ses coreligionnaires ${ }^{6}$. Le vice-chancelier rappelle aussi que, après la guerre de Crimée, le rapport de forces est changé en Orient. Si auparavant les Russes avaient des relations seulement avec "les Turcs", désormais «ils ont affaire aux Européens». Dans ces conditions, selon le chef de la diplomatie impériale, l'ouverture d'une mission à Jérusalem est le seul moyen pour la Russie d'avoir une place importante en Orient. Toutefois, le prince Gortchakov ne parle pas dans son instruction d'une mission diplomatique, comme on pourrait le supposer. Plus étonnant encore, le ministre des Affaires étrangères russe n'évoque jamais dans ce document l'idée de la 
création, même dans un avenir lointain, d'un consulat russe à Jérusalem ${ }^{7}$. Dans ce cas, de quelle mission s'agit-il ? Et surtout dans quel but devrait-elle fondée?

En fait, le prince Gortchakov propose de refonder la mission ecclésiastique russe de Jérusalem, ouverte dans la ville sainte pour la première fois en 1847 et fermée au cours de la guerre de Crimée. Toutefois, selon le chancelier, cette nouvelle mission, fondée "sur de nouvelles bases", devra tout d'abord être «utile à l'Orient» [Vostoku]. Plus précisément, à travers cette mission les Russes devront "prendre soin des trois éléments nationaux [tri narodnykh elementa] - grec, slave et arabe - qui composent l'Orthodoxie», pour que ces «tribus» (plemia) puissent résister «aux tentations malignes de la propagande occidentale ». Comme, selon Gortchakov, « le Russe est aimé par tous - par le Grec et par l'Arabe orthodoxe, et [comme] même les hétérodoxes - les Latins, les Arméniens, les Coptes, les Syriaques et les Chaldéens - se rapprochent facilement » de lui, le devoir de la Russie et, donc, de sa mission ecclésiastique, est d'être "pacificatrice entre les adversaires » et surtout «bienfaitrice des Arabes $»^{8}$. Et comme «le culte des Grecs se trouve en déclin », le vice-chancelier pose que la mission ecclésiastique devra également montrer "aux Franks » et à toutes les confessions présentes en Terre Sainte, à travers la célébration par l'évêque russe du service divin au Saint-Sépulcre, «la meilleure partie de l'Orthodoxie, à savoir l'Orthodoxie russe, complètement inconnue en Orient $»^{9}$. Gortchakov ajoute que "la splendeur de la liturgie orthodoxe est beaucoup plus importante à Jérusalem qu'à Athènes ", car "la Ville sainte est le centre spirituel non seulement de l'Orient, mais aussi de l'Occident $»^{10}$ et, donc, elle représente le « point de rapprochement de toutes les confessions $»^{11}$. Pour toutes ces raisons, selon le ministre des Affaires étrangères russe, la question de la refondation de la Mission ecclésiastique russe à Jérusalem ne peut pas être traitée comme « une question ordinaire» de la politique russe en Orient ${ }^{12}$; pour y réussir, il serait important de ne pas « économiser d'argent, car l'argent est notre seule arme en Orient maintenant » et "personne ne peut nous empêcher de l'utiliser, parce que c'est un moyen qui n'est ni politique ni militaire $»^{13}$.

8 Ce document, rédigé juste après la défaite militaire de la Russie, a eu une grande importance pour la suite de l'histoire politico-religieuse russe mais aussi slave et arabe. En effet, pour la première fois dans l'histoire de l'Empire russe à ce niveau du pouvoir impérial et à l'encontre de l'idéologie conservatrice du règne précédent de Nicolas I ${ }^{\mathrm{er}}$, Gortchakov suggère, pour renforcer la position religieuse russe en Orient, de soutenir non pas le haut clergé grec des Églises orientales, mais les «simples fidèles » arabes, «pauvres, peu instruits et mal traités par les Grecs». Il propose donc de revoir les relations de l'Empire russe, y compris d'ordre financier, avec la hiérarchie orthodoxe des patriarcats orientaux, en particulier avec celui de Jérusalem. Ce faisant, Gortchakov introduit et fixe, dans l'espace impérial russe l'idée de la distinction ethnique au sein de l'œkoumène orthodoxe ; plus largement, il introduit le principe national dans la gestion des affaires religieuses. Quelques années plus tard, ce principe sera mis en pratique à travers les nombreuses tentatives de reconfiguration de l'orthodoxie slave, procheorientale et, finalement russe ; parmi celles-ci figurent la gestion de la " querelle grécobulgare » dans les années 1870, ou l'affaire du premier patriarche arabe d'Antioche (1899), ou encore la question de la construction, entre 1884 et 1896, de l'église Alexandre Nevsky sur le « domaine russe » à Jérusalem, sur laquelle on reviendra plus tard. 
9 Au sein du gouvernement impérial russe, le plan d'action du vice-chancelier Gortchakov n'était pas la seule initiative présentée à l'empereur Alexandre II dans le contexte postérieur à la guerre de Crimée. En effet, en janvier 1856, en plein préparatifs du Congrès de paix de Paris, le grand-duc Constantin, qui se trouvait à la tête de la Marine russe, se préoccupait de préserver une partie de l'effectif de la flotte de la mer Noire. Il proposa à son frère de créer une société privée de navigation en charge d'entretenir « un nombre important de grands bateaux » qui, en cas de nécessité militaire, pourraient être "loués ou achetés par le gouvernement dans le but de transporter des troupes et de transformer ces bateaux en pavillons de guerre $»^{14}$. Dans une autre note rédigée en avril 1856, le grand-duc Constantin souligne que « cette société pourrait remplacer jusqu'à un certain degré notre précédente flotte de la mer Noire et cela pour un moindre coût ». Par ailleurs, cette société " pourrait être utile pour le développement du commerce maritime, en prenant en charge le transport des marchandises russes sur les bateaux russes » et "enfin, grâce à la communication incessante avec les différents points de l'Orient orthodoxe et au transport d'un grand nombre de pèlerins en Palestine et au mont Athos, elle aiderait fortement à notre rapprochement avec nos coreligionnaires et elle contribuerait à obtenir " pour la Russie une place importante "en Orient $»^{15}$. Pour que les premières courses de la Société de navigation ne soient pas déficitaires, il fallait, selon le grand amiral de la flotte impériale, «attirer l'attention de la population» en diffusant l'information « dans toute la Russie sur les conditions pratiques et commodes de la future navigation pour tous les gens désireux de se rendre aux Lieux saints [...] à Jérusalem et au mont Athos ». Pour renforcer l'intérêt du peuple russe à l'égard du mouvement du pèlerinage, il fallait tout simplement rédiger un « Guide du pèlerin orthodoxe aux Lieux saints» avec les informations sur «les trajets, les hôtels et les moyens de communication", ainsi que sur "les prix de l'approvisionnement en produits de première nécessité, des traversées [pereezdy] et sur l'hébergement». Il convenait d'utiliser le modèle des "Guides des voyageurs en Europe ", en l'adaptant toutefois aux besoins de «nos nobles, marchands, bourgeois, religieux, etc. $»^{16}$. Selon le grand-duc Constantin, « cet ouvrage suscitera [en Russie] le désir d'aller [aux Lieux saints] $»^{17}$.

Dans le cas de la proposition du vice-chancelier, comme dans le cas de celle du grandduc, l'objectif était le même - affirmer les positions de la Russie dans l'espace oriental pour renforcer sa position en Europe. Tous deux suggéraient de s'appuyer sur les acteurs religieux, sans toutefois demander l'avis de la hiérarchie ecclésiastique orientale et du Saint-Synode russe. Le premier proposait de s'appuyer sur l'évêque à la tête de la mission ecclésiastique russe à Jérusalem, et le second sur les pèlerins russes désireux d'aller en Terre sainte ou au Mont Athos. Mais si le vice-chancelier voulait changer le rapport des forces à l'intérieur du monde orthodoxe proche-oriental, le grand amiral pensait en premier lieu au sauvetage d'une partie de la flotte russe. Quoi qu'il en soit, ces projets furent mis en œuvre et eurent un impact - immédiat ou sur la longue durée - à Jérusalem et en Russie même.

11 En effet, Alexandre II approuva le projet issu du ministère des Affaires étrangères russe. La mission ecclésiastique de Jérusalem sous l'égide de l'évêque fut refondée le 27 mars 1857 et l'évêque Kirill Naumov arriva dans la ville sainte en mars 1858. L'empereur approuva également le projet issu de la marine russe. La Société russe de navigation à vapeur et de commerce obtint l'autorisation de commencer ses activités dans la mer Noire et au Levant. La première course de la Société, entre Odessa et Constantinople, 
eut lieu le 21 mai 1857. Quelques semaines plus tard, le 4 juillet, l'ancien navire de guerre Chersonèse, transformé en bateau de navigation et de commerce, quitta Odessa pour aller à Marseille, en passant par Constantinople, le mont Athos, Syra et Messine. À bord de ce bateau se trouvait non seulement l'équipe des dirigeants de la Société qui voulaient ouvrir la première ligne de communication maritime entre la Russie et la France, mais aussi B. Mansurov, un fonctionnaire de la Marine envoyé par le grand-duc Constantin en Europe et Orient quelques mois plus tôt pour préparer le guide de pèlerin russe mentionné plus haut.

12 Le séjour de Mansurov à Jérusalem et dans ses alentours entre le 22 janvier/ 9 février et le $5 / 17$ mars $1857^{18}$, ses observations sur les conditions de vie des « simples pèlerins orthodoxes » venus en masse de Grèce, d'Arménie, de Russie ou encore des territoires balkaniques et «mal accueillis» en Terre Sainte par le clergé du patriarcat de Jérusalem, ainsi que ses rencontres en mai 1857 avec N. A. Arkas et N. A. Novosel'skij, pères-fondateurs de la Société de navigation, changèrent la nature du texte qu'il rédigea à Paris pendant l'été et l'automne 1857. En effet, Mansurov explique lui-même que « le résultat de l'étude de la vie de nos pèlerins en Orient » permet de conclure que «ni le gouvernement, ni la Société ne peuvent encore» diffuser parmi le peuple orthodoxe russe « un guide populaire [obtsenarodnyj] ». En effet, « au cas où cet ouvrage rencontrerait le succès ", il " pourrait augmenter le nombre des pèlerins » sur les Lieux saints. Or, précise B. Mansurov, en connaissant l'état de l'accueil et de l'accompagnement des pèlerins orthodoxes en Palestine, "il serait malhonnête » de ne penser qu'aux bénéfices financiers de la Société et de susciter l'intérêt pour le pèlerinage parmi «les gens du peuple ». Ceux-ci ne peuvent pas même imaginer «les épreuves qu'ils devront surmonter au cours du pèlerinage ». En conséquence, à la place du guide, Mansurov rédige et présente le 17/29 décembre 1857 au grand-duc Constantin un rapport intitulé "Les Pèlerins russes en Palestine », dans lequel il propose une vision "pragmatique » des intérêts russes "en Orient » par rapport au pouvoir ottoman, aux puissances européennes et à la hiérarchie grecque des patriarcats orientaux. Il déclare: «Nous devons être plus égoïstes, nous ne devons pas exprimer nos sympathies à tout va [ne rastotchat' uverenj $v$ nachikh simpatiakh], nous ne devons aimer que nous-mêmes et montrer que nous pensons peu aux autres, nous devons tout observer avec beaucoup de vigilance, nous ne devons jamais laisser passer notre chance d'utiliser les fautes des autres, nous devons laisser tomber tout sentimentalisme et les idées d'amour [...], nous ne devons jamais être satisfaits par les choses déjà acquises, mais nous devons toujours aller plus loin » ([Mansurov] $1857: 202$ ).

Plus concrètement, Mansurov reprend l'idée, déjà exprimée par les fondateurs de la Société russe de navigation à vapeur et de commerce, d'ouvrir un poste de consul et d'agent de ladite Société à Jérusalem, dont le but serait de protéger «a) nos intérêts contre les actions arbitraires du gouvernement turc; b) contre les empiétements incessants des puissances occidentales sur notre influence et nos intérêts " (Mansurov 1858, annexe I : 5). Et surtout B. Mansurov propose de créer à Jérusalem « un coin de Russie » [russkij ugol] pour accueillir et accompagner "les simples pèlerins russes » indépendamment du clergé grec du patriarcat de Jérusalem, qui se montrait, selon les observations de Mansurov, beaucoup plus soucieux de ses intérêts financiers que des besoins spirituels des fidèles orthodoxes ${ }^{19}$. Pour construire ce "coin de Russie " à Jérusalem et, plus généralement, pour "prouver la sincérité des sentiments [des Russes] envers l'Orthodoxie et sa gloire ", il était insuffisant de prier. Il "fallait la démontrer par l'action [delo]» et surtout par les moyens financiers (Mansurov 1858 : 
104) offerts non pas par le gouvernement impérial, mais par tous les orthodoxes russes : «Si chaque fidèle des 50000000 orthodoxes offre pour la cause, commune et sacrée, un kopeck, cette misérable aumône apportera annuellement une somme de 500000 roubles, ou 2 millions de francs; mais si chacun donne 2 kopecks, dans ce cas il y aura tout d'un coup une somme d'un million de roubles, ou quatre millions de francs... » (Mansurov 1858 : 105). Autrement dit, il fallait demander au « simple peuple russe " d'aider les "simples pèlerins russes" transportés vers les Lieux saints de Palestine, de Constantinople ou du mont Athos par la Société russe de navigation à vapeur et de commerce, créée sous le patronage du grand amiral et grand-duc Constantin pour sauver la flotte de la mer Noire. Mansurov présente cette Société comme « le meilleur moyen pour la réalisation de tout ce que demandent la dignité et l'intérêt de l'Église russe et de la politique russe", car "les intérêts de notre gouvernement en Orient correspondent parfaitement aux intérêts de la Société » ([Mansurov] $1857: 160$ ).

Les propositions de B. Mansurov, acceptées par le grand-duc, se transforment en décret impérial publié le 27 février 1858, ordonnant des collectes spéciales d'argent dans chaque église de l'empire [kruzhetchnyj sbor] et l'ouverture d'une souscription pour la construction d'hospices [obustrojstvo] pour les pèlerins russes en Palestine. De son côté, la Société russe de navigation à vapeur et de commerce annonce son accord pour la "prise en charge gratuite [bezvozmezdno] de l'acquisition des terres et de la construction des bâtiments nécessaires pour l'accueil des pèlerins ", ainsi que pour « la prise en charge de leur vie » en Palestine ${ }^{20}$.

15 Le Saint-Synode de l'Église orthodoxe russe a exprimé plusieurs fois une opinion assez mitigée sur les collectes d'argent dans les églises de l'Empire "pour les hospices » et les pèlerins en Palestine, et surtout sur la mobilisation en faveur du mouvement de pèlerinage, perçue comme "inopportune » et même "nocive et dangereuse " pour le peuple russe. Pourtant le grand-duc Constantin, soutenu par Alexandre II, décida d'envoyer encore une fois à Jérusalem son bras droit dans les «affaires palestiniennes », B. Mansurov ${ }^{21}$. Ce dernier était accompagné par le premier consul russe à Jérusalem, qui était en même temps l'agent de la Société de navigation et de commerce, V. Dorgobouzhinov. Leur mission était d'acheter les terres nécessaires pour la construction du " "coin" de Russie » à Jérusalem.

16 Six mois après leur arrivée à Jérusalem, le consul russe signe le 20 mars 1859 le premier acte $^{22}$ d'achat des terrains appartenant aux coptes et situés à proximité du SaintSépulcre : "[...] Monsieur Wladimir Dorgoboujinov, Conseiller d'état, gentilhomme de la Chambre, Chevalier et Consul de l'Empire puissant de la Russie à Jérusalem a acheté avec l'argent de l'État puissant de la Russie, et pour cet État, des vendeurs les modèles de la Nation chrétienne le Curé George procureur de la Nation Copte à Jérusalem et son frère Risek, fils de Tomase ... tout le terrain appartenant à eux et en leur jouissance légale, lequel terrain les vendeurs l'ont eu en héritage... Le prix est fixé 122.432 piastres monnaie ayant cours légal... $»^{23}$.

17 Ainsi, par la modalité de cet achat, «le domaine copte» [koptskoe mesto] se transforme en «domaine russe » [russkoe mesto], dont le nettoyage des "déchets centenaires » commence en novembre $1859^{24}$.

18 Le 28 février 1861 V. Dorgobouzhinov, à ce moment-là ancien consul et agent de la Société de navigation à Jérusalem, fait un rapport au grand-duc et au Comité de Palestine, créé sous son patronage en mars 1859, sur « les découvertes archéologiques » 
faites sur le «domaine russe» lors de ce nettoyage. Il souligne qu'« au nord-est du domaine russe se trouvent des ruines des portiques et des propylées appartenant à la basilique du Saint-Sépulcre de Constantin ${ }^{25}$. Il relève l'importance symbolique de la situation géographique du «domaine russe»- «seulement à 50 pas par rapport à l'autel de la cathédrale de la Résurrection»-, l'importance de «la future place de la Russie au sein du monde orthodoxe", déjà "jalousée ", "pas seulement par les confessions hétérodoxes ${ }^{26}$, mais aussi la nécessité de trouver un abri pour les pèlerins russes obligés d'attendre, "dans le froid ", après le service divin nocturne au SaintSépulcre, l'ouverture des portes de Jaffa ; il propose par la suite d'acheter des terrains avoisinant le $«$ domaine russe $»^{27}$.

19 Le but suprême de cet agrandissement est la création sur ce lieu d'un ensemble architectural composé d'un hospice pour les pèlerins, d'« une maison consulaire » et d'« une église [domovoj khram]». Selon V. Dorgobouzhinov, cette église doit être construite sur « les ruines de l'ancienne basilique de l'empereur Constantin », par « la main énergique de l'homme [deiatel']» - à savoir le grand-duc Constantin - qui «a attiré l'attention cordiale » du Souverain sur les problèmes des pèlerins russes en Terre Sainte. Cette construction viendrait ainsi « immortaliser le souvenir de tout ce qui a été fait dernièrement par des Russes à Jérusalem ${ }^{28}$.

Cette idée est très fortement soutenue par le grand-duc Constantin. Ce dernier, après avoir reçu l'autorisation de son frère l'empereur, ordonne le 11 avril 1861 « l'achat des terres auprès de la cathédrale du Saint-Sépulcre à Jérusalem ${ }^{29}$. Toutefois, malgré le soutien du Patriarcat de Jérusalem (ou à cause de son « soutien »?) et malgré les efforts du consul ${ }^{30}$, la Russie ne réussit pas à acquérir toutes les terres si convoitées par plusieurs acteurs locaux et européens ${ }^{31}$, ni à construire à ce moment-là les bâtiments en question.

21 Pourquoi, après avoir investi autant - diplomatiquement, matériellement et même personnellement - dans l'achat du « domaine copte » devenu « russe », les responsables des affaires palestiniennes abandonnent-ils cet endroit si bien situé du point de vue symbolique et stratégique au cœur de Jérusalem? Quelques savants étrangers nonorthodoxes, comme les Français Melchior de Vogüe et Ch. Clermont-Ganneau, ou les Britanniques Ch.-W. Wilson et Conder - obtiennent dans les années 1860-70, à titre personnel ou en qualité de représentants d'institutions étrangères, la permission des diplomates russes de fouiller et de décrire ce lieu. Mais pourquoi est-il ensuite remblayé par les agents russes?

Pour l'instant, on peut proposer deux explications, en partie trouvées dans les sources elles-mêmes: l'une est plus "visible ", rationnelle, d'ordre économico-diplomatique ; l'autre, d'ordre symbolique, est moins perceptible, moins exposée, mais peut-être plus importante.

En effet, en mars 1859, B. Mansurov et V. Drogobuzhinov se lancent non seulement dans l'acquisition de la première partie du « domaine copte " près du Saint-Sépulcre, mais aussi des terrains qui se trouvaient en dehors de la Ville sainte, en premier lieu, sur la place de Meïdan, située «à 10 minutes à pied de la porte de Jaffa et à 15-18 minutes du Saint-Sépulcre $»^{32}$. Ce terrain, acquis en partie par achat, direct ou indirect, en partie grâce à "la concession » obtenue du Sultan ${ }^{33}$ à l'occasion du pèlerinage du grand-duc Constantin en Terre Sainte en mai $1859^{34}$, était situé sur une hauteur permettant «d'embrasser sur un vaste horizon la Transjordanie et les montagnes d'Arabie à Bethléem », et «ayant l'ensemble de Jérusalem à ses pieds $»^{35}$. À 
la différence du « domaine russe " à côté du Saint-Sépulcre, ce terrain, «à cheval sur deux routes » menant à Bethléem, Naplouse, Jaffa et au monastère de la Croix, donnait la possibilité de "construire sur une vaste échelle $»^{36}$. Inspiré par l'ampleur du terrain acquis ${ }^{37}$, B. Mansurov propose, dans un texte rédigé probablement au début de $1860^{38}$, d'y fonder une "nouvelle laure russe " et donc d'y construire une cathédrale pour accueillir 1000 personnes, une maison pour la Mission ecclésiastique, un hospice pour 300 pèlerins-hommes et un autre hospice pour 500 femmes, un hôpital de 60 lits et des bâtiments de service ${ }^{39}$. La " propriété russe " [russkie vladenia] sur la place de Meïdan sera, selon les plans de B. Mansurov, "clôturée par un mur en pierre ", et donc refermée sur elle-même, mais la cathédrale - «le centre et l'élément essentiel de l'ensemble architectural»- faite "sur le modèle [po podobiu] des anciennes cathédrales monastiques dans le style byzantin, préservées seulement au mont Athos ", «sera construite sur une hauteur dominant tout l'espace environnant ${ }^{40}$.

L'achat du terrain sur la place de Meïdan ne requérait pas une somme astronomique, malgré la hausse des prix consécutive à l'arrivée des Russes sur le marché hiérosolymitain ${ }^{41}$. Mais la construction de la future « Nouvelle Jérusalem » à l'extérieur de la « vieille ville $»^{42}$ allait coûter très cher : $B$. Mansurov avait évalué à 755872 roubles (plus de 3 millions de francs) ${ }^{43}$ le coût de ces constructions. Même si le Comité de Palestine, créé en mars 1859, disposait des sommes nécessaires ${ }^{44}$, il est probable que la cherté de la réalisation du projet sur la place de Meïdan causa l'«abandon » du développement $d u$ "domaine russe» près du Saint-Sépulcre. Mais les obstacles financiers ne furent peut-être pas la seule cause de cela et un autre motif de concurrence joua également entre les deux projets.

En effet, les travaux de construction sur la place de Meïdan commencent en 1860 par la pose [zakladka] de la première pierre de la future cathédrale le 30 août, ou le jour de la commémoration du saint Alexandre Nevsky ${ }^{45}$, "patron» du tsar Alexandre II. La proposition de dédier la future église à saint Alexandre Nevsky avait été faite en mai 1860 par le grand-duc Constantin ${ }^{46}$. Néanmoins cette proposition reçut très tôt des objections issues de l'entourage même du grand-duc. Dans une lettre datée du 24 mai 1860 , le prince A. D. Obolenskij, proche du grand-duc et premier directeur du Comité de Palestine, soulignait qu'Alexandre Nevsky « ne se trouve pas parmi les saints de l'Église grecque ». Selon Obolenskij, ce fait pouvait entraîner des difficultés de la part de la hiérarchie grecque. C'est pourquoi il demanda au grand-duc Constantin d'abandonner l'idée de donner à la cathédrale russe le nom d'Alexandre Nevsky et de choisir assez rapidement soit le nom d'un autre saint reconnu par les patriarches orientaux, soit une fête religieuse pour la cérémonie de la pose de la première pierre de l'église qui en tirerait son $\operatorname{nom}^{47}$. C'est ainsi que la cathédrale qui fut finalement construite sur la place de Meïdan et qui domina longtemps tout Jérusalem reçut le nom de la SainteTrinité, et pas celui d'Alexandre Nevsky. Il est possible qu'après cet échec de nommer la nouvelle cathédrale russe d'après le nom d'Alexandre Nevsky, une partie de la famille impériale et peut-être l'empereur Alexandre II se soient montrés réticents à construire près du Saint-Sépulcre, sur «le reste de la basilique de Constantin » fondée sur le lieu de la crucifixion de Jésus, une " église russe Saint Constantin », patron du grand-duc.

Les problèmes d'ordre financier et diplomatique autour de l'achat des terrains supplémentaires près du "domaine russe» furent donc renforcés par cette concurrence symbolique entre les deux projets de construction de deux églises - l'une, près du Saint-Sépulcre, à la mémoire de l'empereur et du saint Constantin et en 
hommage au grand-duc Constantin; l'autre, en dehors de la vieille ville de Jérusalem, sur une hauteur permettant de "dominer" la ville, à la mémoire du saint russe Alexandre Nevsky et en hommage à l'empereur Alexandre II.

Si cette conjonction peut expliquer l'« abandon » du « domaine russe » dans les années 1860-1870, elle n'entame en rien la détermination des Russes à imposer, toujours dans le contexte de tensions, l'orthodoxie russe au cœur du christianisme pour marquer Jérusalem et la Terre sainte.

\section{Temps d'appropriation, discussions et même « sabotage »}

Il fallut attendre encore vingt-cinq ans avant que les acteurs russes impliqués dans les "affaires palestiniennes" ne ressuscitent l'idée «d'étudier scientifiquement» le « domaine russe » situé près du Saint-Sépulcre de façon à pouvoir l'utiliser par la suite. Cette période fut marquée par le changement de règne et de climat politique en Russie à la suite de l'assassinat d'Alexandre II en mars 1881, par le déclin de l'influence du grand-duc Constantin et de son entourage, et surtout par la fondation, en mai 1882, sous le patronage du grand-duc Serge ${ }^{48}$, de la Société orthodoxe de Palestine ${ }^{49}$.

En effet dans un discours prononcé en mars 1883, et intitulé "Les tâches de la recherche scientifique en Terre Sainte ", V. Khitrovo, assistant du grand-duc Serge, brosse un état des lieux des recherches sur la Terre Sainte effectuées par les savants des différentes confessions chrétiennes, depuis des temps très anciens jusqu'aux années 1880 (Khitrovo 1883 : 39-60). Khitrovo distingue deux types de travaux scientifiques consacrés à l'Orient judéo-chrétien : "Le travail qui peut être effectué dans le silence du cabinet et le travail qui doit être réalisé en Terre Sainte» (Khitrovo 1883: 50-51). Selon lui, la Terre Sainte peut être considérée, du point de vue scientifique, comme « un grand musée, un catalogue de connaissances déjà rassemblées, mais qui doit être mis en ordre " (Khitrovo $1883: 51$ ). Ce travail doit être attribué aux savants orthodoxes russes seulement, et non pas aux catholiques ni aux protestants, car la Terre Sainte « appartenait à l'Empire byzantin depuis les premières années du christianisme et [que] l'orthodoxie était, dans cet Empire, comme à la maison " (Khitrovo 1883: 54-55). Le «travail en cabinet» doit être accompagné, selon Khitrovo, par un travail sur place; plus précisément, la Russie doit effectuer des fouilles archéologiques auprès du SaintSépulcre.

Le grand-duc Serge, sous l'influence de ce discours ainsi que d'autres textes de Khitrovo ${ }^{50}$, demande au ministre des Affaires étrangères l'autorisation de rouvrir le chantier sur le "domaine russe ", offre 1000 roubles d'or pour cette affaire et invite l'archimandrite Antoine Kapustine, à la tête de la Mission ecclésiastique depuis 1864, à effectuer ces fouilles, avec l'aide de l'architecte officiel de Jérusalem, C. Schick (Khitrovo 1883: 54-55) ${ }^{51}$. L'ambassadeur russe à Constantinople, A. Nelidov, qui devait assurer le bon déroulement des « recherches scientifiques » de la Société de Palestine au cœur de Jérusalem, conseille au grand-duc de ne pas mettre en avant l'importance politique et religieuse des fouilles, «ce qui n'empêcherait pas cependant de rendre publics les résultats des travaux effectués, après leur achèvement, mais plus encore de tirer tous les profits de cette affaire dans un sens politique, scientifique et religieux $»^{52}$. L'organisation de ces fouilles, et de leur mise en œuvre - ce que représentent une autre modalité d'action russe à Jérusalem - illustrent bien les interactions existantes entre la 
«science orthodoxe », représentée par le Père Kapustine ${ }^{53}$, la diplomatie russe - car le lieu appartient toujours au consulat - et, finalement, le pouvoir impérial qui assure le financement des recherches archéologiques et qui utilise par la suite leurs résultats pour marquer la présence de l'Empire orthodoxe en Palestine.

31 En mai 1883, c'est-à-dire deux mois après le début des fouilles, le Père Kapustine rapporte dans une note aux dirigeants de la Société qu'il a dépensé 495 roubles sur les 1000 offerts par le grand-duc " pour évacuer 13100 sacs de gravats par des ânes " ${ }^{54}$. Il poursuit les fouilles dont les résultats « dépassent » rapidement toutes «les attentes». Dès le 2 janvier 1884 , le grand-duc déclare dans un rescrit au secrétaire du ministère des Affaires étrangères : «Bien que les fouilles ne soient pas encore achevées, elles ont connu un grand succès. Non seulement ont été trouvées des traces des seconds remparts de la Jérusalem moyenne-hébraïque, qui confirment l'authenticité du lieu de sépulture de Jésus, mais on a même découvert sur le domaine russe la porte qui menait de la Ville au Golgotha $»^{55}$. Dans le septième tome du Recueil orthodoxe de Palestine ${ }^{56}$, publié en octobre 1884, l'archimandrite Antoine et les responsables de la Société, s'appuyant sur leurs propres découvertes, fondées en grande partie sur les recherches précédentes des savants étrangers mentionnés plus haut, annoncent officiellement avoir précisé les connaissances concernant la basilique de Constantin, avoir exhumé les traces d'anciennes murailles de Jérusalem (le mur qualifié de « deuxième muraille ») et enfin, avoir découvert « le seuil de la Porte à travers laquelle le Christ est monté au Calvaire » (Les fouilles dans le domaine russe..., 1884-1887 ; Mansurov 1887, p. 9). À travers ces « découvertes" largement diffusées en Russie ${ }^{57}$, mais aussi à l'étranger ${ }^{58}$, la Russie voulait s'approprier symboliquement deux des lieux les plus sacrés du christianisme, à savoir une partie du Chemin de croix qui se trouvait sur le «domaine russe » et qui menait vers le Golgotha, et le Saint-Sépulcre lui-même, qui jouxtait le lieu appartenant à la Russie. Cette appropriation devait être parachevée par la construction d'un «édifice spécial» sur le «domaine russe ». Pour récolter des fonds dans le but de «l'embellissement [blagoustrojstvo] du «domaine russe», la Société impériale orthodoxe de Palestine et les évêques orthodoxes lancèrent, en janvier 1885, un « appel au Peuple russe " (Mansurov 1887 : 12). Les fouilles, les « découvertes archéologiques » et surtout l'idée de la construction d'un «édifice " près du Tombeau de Jésus-Christ, imaginées et mises en œuvre par la famille impériale, par les diplomates et les savants ecclésiastiques, étaient partie intégrante de la politique de la Monarchie russe, à l'intérieur et à l'extérieur des frontières de l'Empire.

En effet, comme le montre Richard Wortman à travers l'analyse de la construction de la cathédrale de la Résurrection à Saint-Pétersbourg sur le lieu d'assassinat d'Alexandre II, le règne d'Alexandre III marque une nouvelle étape dans la construction du mythe monarchique russe (Wortman 2000 : 246). À partir de ce moment, la Résurrection et Jérusalem deviennent " le point de départ de narration sacrée de la Monarchie russe », et où «le Golgotha est transposé directement en Russie » (Wortman $2000: 246)$. Mais, comme le montre l'histoire des fouilles et ses suites, le pouvoir impérial visait aussi à occuper l'espace religieux et politique à Jérusalem et en Terre sainte ainsi qu'à mettre son empreinte sur ces territoires de l'Empire ottoman convoités à cette époque par toutes les grandes puissances européennes et toutes les confessions chrétiennes. Plus encore, ces projets répondaient à la volonté russe de peser sur les rapports de force au sein du monde orthodoxe proche-oriental. 

n'arriva pas tout de suite, après la fin du travail archéologique sur le « domaine russe », à y construire l'« édifice spécial » projeté. Cette fois, il fallut compter avec la résistance à cette idée manifestée par B. Mansurov, qui était à ce moment le directeur de la Commission de Palestine ${ }^{59}$, en concurrence avec la Société de Palestine, et qui décida d'agir, à la fois secrètement et ouvertement, à Jérusalem et à Saint-Pétersbourg. Autrement dit, si en 1861, B. Mansurov soutenait l'idée de construction d'une église russe près du Saint-Sépulcre, en 1884-1885, il avait radicalement changé d'avis.

En effet, à la fin de 1884, Mansurov se rend en Palestine et y reste plusieurs mois. Son but est de préparer une publication sur les fouilles et sur les «découvertes scientifiques » faites par la Société, mais aussi d'entrer en contact avec les principaux protagonistes du projet sur le "domaine russe", notamment avec le consul russe à Jérusalem V. Kozhevnikov. L'analyse des sources inédites donne à penser que B. Mansurov dicte pratiquement les rapports de ce dernier au ministère des Affaires étrangères concernant le « domaine russe » dans le but d'influencer l'opinion de SaintPétersbourg ${ }^{60}$. Par ailleurs, dans une lettre datée du 21 janvier 1885 à son mentor et ancien chef A. V. Golovnine, B. Mansurov explique sa vision des choses : «Pardon pour mon si long silence... Ici, j'ai beaucoup de travail... en plus, il m'est très difficile d'agir dans les conditions où des gens inintelligents et incompétents... me tombent dessus. Heureusement, cette période se termine avec le départ de V. Khitrovo, qui a tout fait pour saboter notre affaire et pour diminuer notre crédit ici. Je ne veux pas vous en écrire davantage, car vous avez lu par vous-même son appel... $»^{61}$. B. Mansurov critique la Société de Palestine car ses responsables veulent construire «quelque chose de peu défini [tcho-to neopredelennoe] $\aleph^{62}$. Selon Mansurov, l'idée de la construction d'un «édifice spécial» à côté du Saint-Sépulcre peut être comparée avec l'idée «du développement [zastrojka] d'un lieu aussi important et aussi sacré que le forum Romain ». "Comment a-t-on pu répondre à quelqu'un qui proposait de faire une construction [nadstrojka] sur le reste de la basilique de Jules César?", demande-t-il à Golovnine. Selon lui, il faut que « les vestiges anciens restent préservés et ouverts pour la science, comme un musée... ${ }^{63}$. Mansurov reste convaincu que son futur ouvrage ${ }^{64}$ va «susciter une tempête d'indignation même au sein de la Société de Palestine, d'autant plus que la négligence, la crédulité et la simple légèreté avec lesquelles a été composé le septième numéro du Recueil de Palestine, sans aucune recherche sur place, dépassent l'imagination ${ }^{65}$.

De retour à Saint-Pétersbourg, B. Mansurov continue son «combat» contre la construction de « l'édifice spécial » sur le « domaine russe » près du Saint-Sépulcre. Il a entre les mains non seulement son premier livre sur les fouilles russes à Jérusalem (Mansurov 1885), mais surtout une « instruction » préparée par A. Golovnine, toujours proche du grand-duc Constantin, qui donne des conseils suivants: "Vous devez personnellement offrir votre livre à l'Empereur, à l'Impératrice et au Tsarévitch, aux grands-ducs Sergej Aleksandroitch, Mikhail Nikolaevitch, Nikolaj Nikolaevitch; envoyer en Crimée un exemplaire au grand-duc Constantin; [...] l'offrir à ....Pobedonostsev ${ }^{66}$; envoyer votre livre dans les rédactions des journaux et revues les plus importants, sans aucune lettre, mais avec une note sur l'enveloppe "de la part de l'éditeur" " ${ }^{67}$.

L'annulation de la pose par V. Khitrovo de la première pierre du futur " édifice spécial » sur le «domaine russe», prévue en 1885, apparaît ainsi comme la conséquence du 
travail du «sabotage » de B. Mansurov et de son « réseau » à Jérusalem et à SaintPétersbourg.

Les textes rédigés dans les années $1885-87^{68}$, dans lesquels Mansurov exprime ses doutes à l'égard des projets de la Société orthodoxe de Palestine, posent une question essentielle: "La Société de Palestine a-t-elle produit des preuves irréfutables que les traces antiques qu'elle a découvertes remontaient à l'époque d'avant Jésus-Christ, au moins au règne d'Hérode le Grand ? » (Mansurov, $1887: 10$ ). Selon lui, il faut répondre à cette question avec assurance et précision " pour ne pas laisser place aux débats et aux doutes, car il s'agit de la reconnaissance de l'authenticité du Saint des Saints chrétien » (Mansurov, 1887: 10). Par ailleurs, Mansurov insiste sur la nécessité de demander à l'Église orthodoxe sa position sur cette question, exprimée autrement que par la simple présence de plusieurs ecclésiastiques dans les instances de la Société de Palestine. Les écrits de Mansurov provoquent une discussion parmi les " palestinologues » mais aussi, plus largement, dans le milieu des archéologues et des historiens russes. Le grand-duc Serge lui-même demande une expertise à la Société savante d'archéologie ("Rescrit du grand-duc Serge" 1884-1887). Sans entrer dans les discussions scientifiques, qui sont l'objet d'une recherche à part, il faudra se demander pourquoi Mansurov, engagé dans "les affaires palestiniennes" depuis 1857, s'est montré si réticent envers les «nouvelles découvertes russes ». Sa vision profondément religieuse, différente de celle de certains membres de la Société de Palestine - y compris peut-être de Khitrovo l'empêchait-elle de mettre les intérêts politiques avant les convictions religieuses ? Ou bien, ses intérêts personnels en qualité de directeur de la Commission de Palestine, mise à l'écart par Khitrovo et le grand-duc Serge, après la création de la Société de Palestine, se cachaient-ils derrière cette position?

Ces questions avaient été déjà posées, en leur temps, par M. Stepanov, un des hauts responsables de la Société de Palestine, au directeur du département asiatique du ministère des Affaires étrangères Zinoviev, dans une lettre du 21 novembre 1885. Après avoir étudié toutes les causes possibles de la résistance de B. Mansurov à la construction sur le « domaine russe »- « l'apolitisme ${ }^{69}$ de l'édifice russe près du SaintSépulcre ", « la jalousie et les soupçons de la part du pouvoir ottoman, des catholiques et du patriarche Nicodème", le travail archéologique inachevé sur le "domaine russe », les conclusions erronées de Schick et « le plan de la Société de Palestine loin de la réalité du lieu »-, Stepanov juge qu'il ne s'agissait que de "l'amour propre » [litchnoe samolubie] de Mansurov et de son attitude hostile envers toutes les activités de la Société : «Mais il faudra se demander : peut-on admettre qu'à cause de la vanité d'une personne, l'idée du grand-duc, de toute la Société et d'un grand nombre de gens en Russie qui ont répondu à notre appel, tombe dans l'oubli ? $»^{70}$.

Cette déclaration de $\mathrm{M}$. Stepanov, derrière lequel se trouvait en réalité le grand-duc Serge et probablement Alexandre III, montre qu'une nouvelle génération d'acteurs russes engagés en Palestine à partir des années 1880 à travers la Société de Palestine va aller jusqu'au bout pour imposer leur idée de la présence russe en Palestine et pour construire, «leur " église près du Saint-Sépulcre, à savoir l'église Saint-Alexandre Nevsky. 


\section{Temps de construction et de reconfiguration} Palestine s'inspirent de l'ancienne idée de V. Dorgobuzhinov de bâtir l'église de saint
Constantin et songent donc à construire une église sur le «domaine russe ». Dans les années 1886-87, ils mentionnent l'idée, sans se référer aux écrits de B. Mansurov, d'y créer « un musée ouvert " ${ }^{71}$. Pendant l'été de 1887, ils reviennent à l'ancien projet de V. Dorgobuzhinov de construire quelques chambres pour les pèlerins sortant du SaintSépulcre après le service orthodoxe nocturne, en ajoutant qu'un autre édifice préservera les ruines antiques et, avec le temps, sera transformé en "musée des Antiquités palestiniennes $»^{72}$. Tout à la fin des années 1880 , les dirigeants de la Société de Palestine définissent l'édifice comme « une nouvelle maison ${ }^{73}$. Mais au début des années 1890, ils parlent presqu'ouvertement de l'église construite, mais «pas encore consacrée ». Le tsarévitch Nicolas, en tournée officielle "en Orient » [po Vostoku] en 1890, devait demander personnellement au Sultan « l'accord pour la consécration de l'église sur "le domaine russe" près du Saint-Sépulcre $»^{74}$, mais son voyage à Constantinople fut annulé. Le 27 novembre 1894, quelques jours après la mort d'Alexandre III, la Société de Palestine propose de "créer un monument éternel [vetchnyj pamaitnik] à la mémoire de l'Empereur défunt, fondateur de la Société, autrement dit, de « fonder [ustroit'] tout près des plus importants Lieux saints chrétiens l'église du saint prince Alexandre Nevsky » ${ }^{75}$. Cette proposition fut soutenue en janvier 1895 par le grand-duc Serge, le chef de la Société ; selon ce dernier, afin de « remplir le devoir de mémoire » envers l'empereur Alexandre III, il faut «consacrer l'église de la Maison russe à Jérusalem à saint Alexandre Nevsky ${ }^{76}$.

Le pouvoir ottoman, selon ses propres règles établies dans le domaine de la construction des édifices religieux, éducatifs et charitables, ne permettait pas aux diplomates russes de bâtir près du Saint-Sépulcre «des églises, des hôpitaux, des écoles » sans le firman du Sultan; en réalité, il était bien informé quant à la nature des travaux effectués sur « le domaine russe ». Le représentant de la Société de Palestine à Jérusalem écrit en octobre 1890 à Saint-Pétersbourg à ce propos : « Notre construction sur le lieu de fouilles suscite des bruits dans la ville. [...] Rechad Pacha, parlant de notre construction sur ce lieu, ne la nomme autrement que "Votre nouvelle église". Donc, cela montre que les pouvoirs turcs sont depuis longtemps au courant que nous sommes en train de construire là-bas une église, et ils le perçoivent avec beaucoup de calme $[. ..] »^{77}$.

Toutefois, ce « calme » était trompeur, car les fouilles et les travaux de construction ont été mal perçus et mal vécus par le patriarcat de Jérusalem. Bien que la Russie se soit présentée comme la protectrice des orthodoxes de l'Empire ottoman, les intérêts économiques, politiques et spirituels du haut clergé grec et des agents russes en Palestine et Syrie étaient différents. Si le rôle de la hiérarchie orthodoxe grecque dans les affaires d'achat des terres supplémentaires à côté du domaine russe entre 1861 et 1864 n'a pas été très clair, comme je l'ai mentionné plus haut, et si les représentants du patriarcat n'exprimaient pas ouvertement leur désaccord avec les actions des agents 
russes lors des fouilles sur «le domaine russe » dans les années 1883-1884, en août 1895 , le patriarche Gérasimos décide de transmettre à travers l'ambassadeur russe à Constantinople A. Nelidov un mémorandum à l'Empereur Nicolas II et une lettre au procureur général K. Pobedonostsev.

$\mathrm{Au}$ début de son mémorandum, le patriarche, rappelle des règles canoniques des Conciles œcuméniques, selon lesquelles il est interdit de fonder des monastères et des maisons de prière sans autorisation de l'évêque de ville; il rappelle également les autorisations déjà données dans le passé à la demande du Saint-Synode de construire sur les territoires canonique de l'Église de Sion des églises russes, même si, selon le patriarche, « dans la majorité des cas, ces églises ne satisfont ni les besoins spirituels ni les besoins matériels $~^{78}$. Dans le cas de la construction de "l'édifice " russe près du Saint-Sépulcre, ajoute-t-il, ces demandes n'ont pas été faites. Qui plus est, après avoir présenté au pouvoir ottoman cet édifice sous différents noms, les hommes d'action de la Société de Palestine ont décidé de le transformer en "église Saint-Alexandre Nevsky $»^{79}$. Leur but, selon le patriarche, est très simple : «il ne s'agit pas en réalité de construire une église domicile en hommage à l'Empereur, mais il s'agit, grâce aux mensonges et à l'encontre des règles ecclésiastiques, de bâtir une splendide cathédrale, un nouveau Lieu saint [sviatynia], un autre centre, près du Saint-Sépulcre, sur le territoire du patriarcat lui-même $»^{80}$. Ainsi, d'après lui, cette construction représente-telle une partie «du programme secret de la lutte de la Société de Palestine contre l'Église orthodoxe palestinienne » dont l'objectif suprême est "de créer un nouveau pouvoir spirituel au sein de notre Sainte Église, de détruire le pouvoir déjà existant et de gérer les affaires de la Terre sainte pour enfin fonder la nouvelle Église et agir sans l'accord d'évêque $\star^{81}$. La preuve en est que les agents de la Société de Palestine déclarent ouvertement vouloir « acheter chez les Abyssiniens le pouvoir sur l'église des saints Constantin et Hélène et créer un passage direct entre la basilique de la "nouvelle maison russe » et la basilique du Saint-Sépulcre ${ }^{82}$. Donc, " » au lieu de rechercher l'union avec l'Église-mère, la Société de Palestine fait tout pour rompre tous les liens avec elle $»^{83}$.

45 A la fin de son Mémorandum, le patriarche demande à l'Empereur Nicolas II de « définir les vraies [pravil'nye] limites de la Société de Palestine ", et déclare que « la Sainte Église ne peut pas reconnaître comme telle l'église bâtie sans autorisation canonique ${ }^{84}$.

Après avoir reçu ce Mémorandum, le ministère des Affaires étrangères russe décide de ne pas répondre au patriarche, car il «ne considère pas possible de présenter officiellement ce document à Sa Majesté $»^{85}$. C'est K. Pobedonostsev, procureur-général du Saint-Synode, en personne qui répond après 18 jours du silence, en accusant à son tour le patriarche « d'accuser toute la Société entière de mentir et de mener une lutte incessante contre la Sainte Église », une "Société impériale gérée directement par le grand-duc Serge et par le Souverain lui-même $»^{86}$. On peut dire que les acteurs russes le ministre des Affaires étrangères, le procureur général du Saint-Synode et les responsables de la Société de Palestine -, en refusant d'accepter le mémorandum, ont, en réalité, montré que la résistance du Patriarche à la construction de l'église russe près du Saint-Sépulcre comme une partie de la politique russe de la reconfiguration du monde orthodoxe dans l'espace ottoman était vouée à l'échec.

Le patriarche, étonné et même affecté par la non-réception diplomatique et par la lettre de K. Pobedonostsev, se trouve sous la pression d'une partie de son propre synode. Malgré ses tentatives de protester et d'imposer ses propres conditions, le 
patriarche n'était pas de taille à s'opposer à la Russie, car cette dernière contrôlait une partie des revenus du patriarcat. Il dut donc finalement donner son autorisation à la consécration de la nouvelle église russe. Le 22 mai 1896, lendemain de la fête des saints Hélène et Constantin, le patriarche Gérasimos consacre la nouvelle église russe SaintAlexandre Nevsky, construite en mémoire d'Alexandre III, fondateur de la Société impériale orthodoxe de Palestine, sur le terrain près du Saint-Sépulcre acheté 37 ans plutôt par les représentants d'Alexandre II. Elle devient une des nombreuses églises Saint-Alexandre Nevsky, érigées à l'époque de la « russification » de la monarchie russe sur le territoire de l'Empire et à l'extérieur de ses frontières ${ }^{87}$. La cérémonie de consécration d'une nouvelle église a été accompagnée par le son des cloches de la cathédrale russe Sante-Trinité, projetée initialement en 1860 comme la cathédrale Saint-Alexandre Nevsky...

\section{En guise de conclusion}

La transformation du "domaine copte ", situé près du Saint-Sépulcre s'opère en plusieurs étapes, à travers plusieurs actions d'ordre économique, politique ou symbolique et dans le contexte de tensions permanentes entre plusieurs acteurs russes et ottomans (en particulier grecs) :

- dans les années 1859-1864, achat de ce lieu par les Russes et projet abandonné de la construction sur «les reliques de la basilique de Saint-Constantin» d'un ensemble architectural russe avec l'église de Saint-Constantin, en hommage à l'activité du grand-duc Constantin à Jérusalem ;

- dans les années 1880, organisation des fouilles archéologiques et discussions autour de leurs « découvertes",

- enfin, dans les années 1890, construction de l'église Saint-Alexandre Nevsky.

L'histoire de cette transformation permet de saisir des changements produits au cours des années 1859-1896 dans les relations entre les acteurs étatiques et religieux de l'espace russe et de l'espace ottoman, en particulier entre les représentants du pouvoir impérial russe et les représentants de l'Église orientale.

On observe, tout d'abord, la transposition de la Russie vers les territoires canoniques des patriarcats orientaux du modèle des relations établies depuis longtemps déjà entre le pouvoir impérial et les religions sur le sol russe. En effet, la proposition du grand-duc Constantin, ministre de la Marine, d'utiliser les pèlerins orthodoxes voulant se rendre en Terre Sainte, au mont Athos ou encore à Constantinople, pour soutenir économiquement la Société de navigation et de commerce, créée après le traité de Paris et la neutralisation de la flotte de la mer Noire, montre bien l'attitude « utilitaire » de la monarchie russe envers l'Église orthodoxe et ses fidèles, sujets du tsar ou du sultan.

51 Ensuite, cette attitude se manifeste à travers les tentatives d'appropriation par la Russie des symboles du christianisme, le Saint-Sépulcre compris, mais aussi à travers l'utilisation des églises russes construites sur la place de Meïdan ou près du Tombeau de Jésus. Ces églises permettent à la Russie de « dominer » symboliquement Jérusalem et, donc, de renforcer politiquement sa position dans l'espace ottoman en face du pouvoir local et des grandes puissances.

52 Enfin, on remarque aussi la transformation de l'idée de la protection par la Russie orthodoxe de ses coreligionnaires de l'Empire ottoman: en effet, après la guerre de 
Crimée, l'Empire russe continue à protéger les chrétiens orthodoxes, slaves et arabes, à travers des actions diplomatiques auprès du pouvoir du Sultan et des consuls européens, mais, comme le montre l'histoire de la transformation du « domaine copte » en église russe Alexandre-Nevsky, ainsi que plusieurs d'autres cas, à partir des années 1860, la Russie commence à contrôler et, à partir des années 1880-1890, même à s'ingérer dans les affaires proprement ecclésiastiques de patriarcats orientaux dans le but de reconfigurer le monde orthodoxe proche-oriental: même si les responsables russes n'ont pas réussi à changer le rapport de force à l'intérieur du Patriarcat de Jérusalem, l'élection du premier patriarche arabe d'Antioche, en 1899, en opposition à la hiérarchie grecque représente un résultat tangible de la politique russe d'ingérence au sein des Églises orthodoxes orientales.

\section{BIBLIOGRAPHIE}

"Les fouilles dans le domaine russe, près du Saint-Sépulcre, effectuées par l'archimandrite Antoine”, Recueil orthodoxe de Palestine, Saint-Pétersbourg, 1884-1887, VII, pp. 1-38 (en russe).

"Rescrit du grand-duc Serge au président de la Société russe d'archéologie", Recueil orthodoxe de Palestine, Saint-Pétersbourg, 1884-1887, VII, pp. 157 ss (en russe).

Astafieva, Elena (2013), "La Société impériale orthodoxe de Palestine : entre savoir, pouvoir, concurrence, 1882-1917”, in Voisinages fragiles : Relations interconfessionnelles dans le Sud-Est européen et la Méditerranée orientale 1854-1923, Paris, Athènes, École française d'Athènes, pp. 121-134.

Astafieva, Elena (2016). “L’Empire russe en Palestine, 1847-1917 : aux origines de la politique russe au Proche-Orient”, Tepsis Papers 10. URL : https://tepsis.hypotheses.org/3187

Astafieva, Elena (2007). “ « Le Bulletin de la Société Impériale Orthodoxe de Palestine et la présence russe en Terre Sainte, 1882-1917”, Cahiers Alberto Benveniste, pp. 121-136.

Fairey, Jack (2012). “'Discord and Confusion... under the Pretext of Religion': European Diplomacy and the Limits of Orthodox Ecclesiastical Authority in the Eastern Mediterranean”, The International History Review, 34 (1), pp. 19-44.

Hopwood, Derek (1969). The Russian Presence in Syria and Palestina. Church and Politics in the Near East, Oxford.

Iakuchev, Mikhail (2013). Les Patriarcats d'Antioche et de Jérusalem dans la politique de l'Empire russe, 1830-début du XXe siècle, Moscou (en russe).

Khitrovo, Vassilij (1883). “Les tâches de la recherche scientifique en Terre Sainte”, Rapport de la Société Orthodoxe de Palestine, années 1882-1883, Saint-Pétersbourg (en russe).

Laurens, H. (1999). La Question de Palestine, I. 1799-1922. L'invention de la Terre sainte, Paris, Fayard. Lissovoj, Nikolaj (2006). La Présence religieuse et politique russe en Terre sainte et au Proche-Orient au $X I X^{e}$-début du XX $X^{e}$ siècle, Moscou (en russe). 
Mansurov, Boris (1887). Les fouilles archéologiques dans la Ville sainte de Jérusalem devant le jugement de la Société d'Archéologie, Riga (en russe).

Mansurov, Boris (1885). La basilique de l'empereur Constantin, Saint-Pétersbourg (en russe).

Mansurov, Boris (1858). Les Pèlerins russes en Palestine, Saint-Pétersbourg (en russe).

[Mansurov, Boris] (1857). Les Pèlerins russes en Palestine, Saint-Pétersbourg (en russe).

Stepanov, D. (2011). "La création de la Société russe de navigation à vapeur et de commerce (1856-1857)”, Vestnik Tcheliabinskogo gosudarstvennogo universiteta, 22 (237), pp. 30-38 (en russe).

Stavrou, Theophanis (1963). Russian Interests in Palestina, 1882-1914, Thessalonique.

Ure, Pinar (2014). Byzantine heritage, archeology, and politics between Russia and the Ottoman Empire: Russian Archeological Institute in Constantinople (1894-1914), dissertation for the degree of Doctor of Philosophy, London.

Vakh, Kirill (2014). “Avant-propos à l'édition”, in Lettres de B.P. Mansurov de ses voyages en Orient orthodoxe, 1857, Moscou, p. 33 (en russe).

Vakh, Kirill (2012). "Le projet hiérosolymitain du grand-duc Constantin Nikolaevitch", in Le Grand-Duc Constantin Nikolaevitch et le pèlerinage russe en Terre sainte, Moscou, pp. 10-111 (en russe).

Vakh, Kirill (2012). Le Grand-Duc Constantin Nikolaevitch et le pèlerinage russe en Terre Sainte, Moscou (en russe).

Vovchenko, Denis (2013). “Creating Arab Nationalism? Russia and Greece in Ottoman Syria and Palestine (1840-1909)”, Middle East Studies, 49 (6), pp. 901-918.

Wortman, Richard (2000). Scenarios of power. Myth and Ceremony in Russian Monarchy, II, Princeton, New Jersey.

Zelenina, Iana, Belik, Zhanna (2011). Les premières cathédrales russes à Jérusalem, Moscou (en russe).

\section{NOTES}

1. C'est grâce au fonds de préfiguration du Labex Tepsis que j'ai pu effectuer mes recherches en Russie pour ce travail, pendant l'été 2015 , et trouver les nouvelles sources d'archives que j'utilise dans cet article. Je remercie Olivier Bouquet, Marc Aymes, Ozgür Türesay pour les discussions stimulantes tenues avant, pendant et après le colloque "Transfaires impériaux: Turquie, Russie » du 15 avril 2015.Un grand merci également à Oleg Anisimov, à Anatole Lozowski, Michel Tissier, Antoinette Guise-Catelnuovo et à Wladimir Berelowitch pour leurs aide, commentaires et conseils précieux. Je voudrais aussi remercier les deux relecteurs anonymes de mon texte, leurs commentaires et remarques étaient et restent très utiles pour cette recherche et celles à venir.

2. La publication récente qui illustre le mieux cette tendance est celle de N.N. Lissovoj (2006).

3. Pour l'instant, malheureusement, je n'ai pas pu effectuer des recherches en Turquie pour compléter les sources trouvées dans les archives russes par les sources des archives impériales ottomanes; mais j'espère que le futur travail dans ces archives me permettra de croiser les sources en différentes langues, de « donner la voix » aux acteurs de l'Empire ottoman, impliqués dans cette histoire, et donc, d'approfondir certaines des questions abordées dans cet article.

4. Rossijskaia Gossudarstvennaia Biblioteka-RGB [Bibliothèque d'État de Russie], Départements des manuscrits, F. 148, Carton 4, D. 12, p. 1-11 (verso).

5. Ibid., p. 1 .

6. Ibid., p. 2 . 
7. Selon le chancelier, avoir « un vice-consul à Jaffa (à condition qu'il ne soit pas Grec) est déjà suffisant ». Ibid., p. 2.

8. Ibid., p. 3.

9. Ibid., p. 3 (verso).

10. Ibid., p. 5 (verso).

11. Ibid., p. 11 (verso).

12. Ibid., p. 11 (verso).

13. Ibid., p. 6 (verso).

14. Rossijskij Gosudarstvennyj Istoritcheskij Arkhiv - RGIA [Archives historiques d'État de Russie], F. 107, op. 1, D. 14, p. 1. Voir aussi Stepanov 2011.

15. Ibid. p. 3.

16. Rossijskij Gosudarsvennyj Arkhiv Voenno-Morskogo Flota - RGA VMF [Archives de la Marine russe],

F. 410, Op. 2, D. 13, p. 3-4, cité par Vakh 2014, p. 33.

17. Ibid.

18. Les lettres de Mansurov à son père, rédigées en français, se trouvent au Gosudarstvennyj Arkhiv Rossijskoj Federatsii - GARF [Archives d'État de la Fédération de Russie], f. 990, op. 1, D.31-33. Voir sa traduction en russe: Vakh 2014. Sur les activités de B. Mansurov et du grand-duc Constantin dans les années 1857-1864, voir Vakh 2011 et 2012.

19. Toutefois, B. Mansurov, après avoir analysé « les sources financières » du haut clergé grec du Patriarcat de Jérusalem, souligne que « la participation du peuple russe à la cause du soutien du Tombeau de Jésus-Christ » n'est pas si importante et ne représente que la neuvième partie des recettes annuelles. Mansurov, 1858, Annexe III p. 16.

20. Rossijskaia natsional'naia Biblioteka - RNB [Bibliothèque nationale de Russie], Département des manuscrits, F.253, op. 1, D. 40, pp. 82-83.

21. Sur les impressions de voyages de B. Mansurov, voir RGAF, F. 990, op. 1, D. 32, pp. 18ss.

22. Il s'agit du texte rédigé en français par Nassoluy-Zadi Es-scial Ibrahim Fuad, « le très humble juge de la ville sainte de Jérusalem», dont on a gardé les particularités stylistiques et grammaticales.

23. RNB, Département des manuscrits, F.253, op. 1, D.40, pp. 210-211(verso).

24. Selon le consul de l'époque, le premier nettoyage a duré plusieurs mois et n'était toujours pas fini en juillet 1860 (probablement le moment de son départ). Ce nettoyage a coûté à la Russie plus de 120000 piastres : "Les 80 ânes ont travaillé en même temps, en faisant 13 aller-retour chaque jour [...]». Le consul explique, en entrant dans les détails de l'histoire de Jérusalem et de sa topographie, que «cette ville a été construite et continue à être construite sur les ruines et les déchets ». Cette "particularité de Jérusalem permet de comprendre, selon V. Dorgobouzhinov, pourquoi l'achat d'un terrain, ou plutôt de sa couche supérieure, ne signifie pas encore un « vrai achat du terrain avec toutes ses profondeurs ». Cela explique aussi les actes arbitraires autour de l'acquisition des terrains à Jérusalem où le pouvoir est corrompu. Le nettoyage du terrain jusqu'à « la terre » est le seul moyen à Jérusalem de « le posséder réellement ». Selon B. Mansurov, entre juillet 1859 et septembre 1860, les Russes ont dépensé 6641 roubles.

25. RNB, Département des manuscrits, F. 253, op. 1, D. 40, p. 279.

26. Ibid., p. 280.

27. Il s'agit de 12 boutiques et d'une boulangerie. Selon l'ancien consul, l'acquisition de 12 boutiques coûterait environ 100000 piastres et celle de la boulangerie beaucoup plus cher, entre 350 et 400000 piastres, car le propriétaire « attend l'arrivée des Russes » et a déjà refusé de vendre son terrain pour 150000 piastres ».

28. Ibid., p. 282 (verso).

29. Constantin a accordé la somme de 23000 roubles pour tous les achats.

30. Le 18 septembre 1861, le consul Sokolov demande conseil à l'évêque Kirill Naumov, chef de la Mission ecclésiastique russe à Jérusalem à ce moment, à propos de la stratégie à adopter dans 
l'affaire d'achat des terrains en question : « [...] soit trouver quelqu'un sur place pour qu'il puisse négocier en notre nom avec les différents propriétaires et donc acquérir des terrains, si possible, hâtivement et secrètement ; soit s'en ouvrir sans rien dissimuler [vo vsem priznat'sia] au Patriarcat grec et demander son soutien amical en toute simplicité [priamoduchno]». Dans un cas comme dans l'autre, souligne Sokolov, les agents russes peuvent avoir des «surprises». S'ils avaient recours à un intermédiaire local, les Russes pourraient être trompés par ce dernier et par les vendeurs. En cas de demande d'aide au Patriarche, «le Patriarcat pourrait acheter tous les terrains bien avant nous ». Malgré ces craintes, les représentants russes à Jérusalem décident de s'adresser au Patriarche, qui leur promet son soutien. En l'état actuel de nos recherches, on ne peut pas dire dans quelle mesure l'aide du Patriarcat a été efficace ou si, au contraire, il aurait bloqué les acquisitions russes.

31. La boulangerie a été achetée pour une somme de 246310 piastres, selon Sokolov (ou selon Mansurov, 13304 roubles); le prix des 11 boutiques a monté jusqu'à 500000 piastres (plus de 25000 roubles); mais même à ce prix, il était impossible de les acquérir, car le gouvernement local l'interdisait aux diplomates russes.

32. RGB, Département des manuscrits, F. 148, Carton 4, D. 13, p. 19.

33. RNB, Département des manuscrits, F. 253, op. 1, D. 40, pp. 213-215.

34. Sur le pèlerinage voir : RGB, Département des manuscrits, F. 148, op. 1, carton 4, D. 6.

35. RGB, Département des manuscrits, F. 148, Carton 4, D. 13, p. 9.

36. RNB, Départements des manuscrits, F. 253, op. 1, D. 40, p. 214.

37. Il s'agissait de 15.799 sazhen' carrés, ou 71 880,5 mètres carrés.

38. Ce projet a été corrigé par le père Léonid (Kavelin), futur chef de la Mission ecclésiastique de Jérusalem, impliqué dans les « affaires palestiniennes » bien avant de sa nomination.

39. RGB, Département des manuscrits, F. 148, Carton 4, D. 13, p. 18.

40. Ibid., p. 20.

41. On trouve l'information concernant cette hausse des prix dans plusieurs documents, notamment dans la correspondance diplomatique. On sait aussi que le consul a dû offrir au Surréyach-Pacha un bakchich d'un montant de 76000 piastres en échange de son aide dans les affaires d'achat et de concession des terrains convoités par les agents russes.

42. Ce nom (Nouvelle Jérusalem) est donné par les habitants arabes aux constructions russes. Cité par Zelenina Belik 2011, p. 35.

43. RGB, Département des manuscrits, F. 148, op. 1, carton 4, D. 13, p. 25.

44. Mansurov parle de 818623 roubles et 37 kopeks que le Comité de Palestine doit récolter vers 1863. Ibid. p. 23. On reviendra sur ces questions dans des recherches ultérieures.

45. L'Église orthodoxe a deux fêtes liées à la mémoire d'Alexandre Nevsky: le 23 novembre (6 décembre), jour de son enterrement, et le 30 août (12 septembre), jour du transfert de ses reliques de Vladimir à Saint-Pétersbourg par Pierre le Grand en 1723.

46. RNB, Département des manuscrits, F. 253, op. 1, D. 40, p. 51.

47. Ibid., p. 51.

48. Il s'agit du fils d'Alexandre II et du neveu d'Alexandre III.

49. Cette Société devient impériale en 1889.

50. Voir, par exemple, sa note datée de novembre 1882 : Arxiv vnechnej politiki Rossijskoj Imperii AVP RI [Archives de la politique extérieure de l'Empire russe], f. 337/2, op. 873/1 (1879-1917), D. 593 «Les fouilles sur le "domaine russe", près du Saint-Sépulcre, à Jérusalem », pp. 1-3.

51. AVP RI, f. 337/2, op. 873/1, D. 593, p. 4.

52. AVP RI, f. 337/2, op. 873/1, D. 593, p. 18.

53. Sur le père Kapustin, voir : Kern 1934 et 1997.

54. AVP RI, f. 337/2, op. 873/1, D. 593, p. 44.

55. Ibid., pp. 54-55. 
56. Il s'agit d'un des organes de presse de la Société orthodoxe de Palestine ; le Recueil de Palestine a été créé en 1881, quelques mois avant la fondation officielle de la Société. Voir plus sur la presse de la Société de Palestine : Astafieva 2007.

57. Plusieurs journaux et revues ont consacré des articles à ces découvertes, comme par exemple le Journal du Ministère de l'Instruction publique, ou la revue Lectures chrétiennes, sans parler des nombreuses publications de la Société, dont le Bulletin de la Société orthodoxe de Palestine, son organe officiel.

58. Les résultats des fouilles furent discutés dans le tome VIII du Zeitschrift des Deutschen PalästinaVereins, notamment dans l'article d'un des acteurs de ces fouilles, C. Schick.

59. Il s'agit de l'ancien Comité de Palestine, transformé en 1864 en Commission.

60. RNB, Département des manuscrits, D. 243, op. 1, D. 43, pp. 3-11 (verso). L'histoire de cette "influence» a été rapportée à I. Zinoviev, chef du consul Kozhevnikov, par les hauts responsables de la Société de Palestine.

61. GARF, F. 990, op. 1, D.9, p. 1. Selon B. Mansurov, les « 2000 roubles » utilisés par la Société de Palestine pour l'organisation des fouilles représentent une petite somme en comparaison avec 31000 roubles, dépensées par lui et Dorgobuzhinov à la fin des années 1850 pour l'achat et le nettoyage du terrain à côté du Saint-Sépulcre. Dans les papiers officiels de la Société, il s'agissait d'une somme de 1000 roubles.

62. Ibid, p. 9.

63. Ibid., p. 9.

64. B. Mansurov voulut publier son ouvrage chez M. Katkov, l'éditeur le plus influent à cette époque en Russie. Au cas où cela aurait été impossible, Mansurov envisageait même de publier son livre à l'étranger. Ibid. p. 9.

65. Ibid., p. 9.

66. Il s'agit du procureur-général du Saint-Synode de l'Eglise orthodoxe russe.

67. RGAF, F. 990, op. 1, D. 241, p. 8 .

68. En plus de son ouvrage principal, publié en 1885, B. Mansurov rédige de nombreux textes consacrés aux fouilles archéologiques russes, parmi lesquels il faut mentionner celui-ci: Mansurov, 1887.

69. Pour l'instant je ne peux pas expliquer le sens du mot «apolitisme», utilisé par M. Stepanov dans ce contexte.

70. RNB, Départements des manuscrits, F. 253, op. 1, D. 43, p.11.

71. RNB, Département des manuscrits, F. 253, op.1, D. 42, p. 36 (verso).

72. AVP RI, F. 337/2, op. 873/1, D.20, p. 70 ; NRB, Département des manuscrits, F. 253, op.1, D. 42, p. 36 (verso).

73. RNB, Département des manuscrits, F. 253, op.1, D. 42, p. 36 (verso).

74. AVP RI, F.337/2, op. 873/1, D. 496, p. 37.

75. RNB, Département des manuscrits, F. 253, op.1, D. 42, p. 36.

76. Ibid., p. 44.

77. AVP RI, F. 337/2, op. 873/1, D. 496, p. 28.

78. NRB, Département des manuscrits, op. 1, F. 253, D. 42, p. 35 (verso).

79. Ibid.

80. Ibid., p. 38.

81. Ibid.

82. Ibid., F. 233, D. 42, p. 40.

83. Ibid.

84. Ibid., p. 42.

85. Ibid., p. 65.

86. RNB, Départements des manuscrits, F. 253, op. 1, D. 42, pp. 151-153 (verso). 
87. Des églises dédiées à Saint-Alexandre Nevsky ont été construites à Paris, Belgrade, Sophia, Copenhague, Varsovie, Revel et d'autres villes. Voir à ce propos : Wortman 2000, II, pp. 248 ss.

\section{RÉSUMÉS}

Cet article est consacré à l'analyse des activités des acteurs russes menées à Jérusalem après la guerre de Crimée. Il présente le cas particulier de la transformation par les Russes d'un espace «domaine copte »- situé au cœur de Jérusalem, près du Saint-Sépulcre, et il montre ainsi que cette transformation s'opère en trois temps en lien avec trois types d'action: le temps de la fondation des institutions russes "en Orient " et de l'acquisition des terres à Jérusalem (1857-1864) ; le temps d'organisation des fouilles archéologiques et de l'appropriation par la Russie des principaux symboles du christianisme (1882-1884); et enfin, le temps de la construction de l'église orthodoxe russe Saint-Alexandre Nevsky près du Saint-Sépulcre et de la reconfiguration de l'orthodoxie proche-orientale en marche (1885-1896...).

The article offers an overview of Russia's activities in Jerusalem in the wake of the Crimean War. Specifically, it examines the case of the so-called "domaine copte" in the heart of Jerusalem near the Holy Sepulchre, which became an object of transformation by Russians. There were three distinct stages of transformation of this symbolically and politically important space, and in the article they are considered from within three distinct contexts: the establishment of Russian institutions "in the East" and the process of land acquisition in Jerusalem (1857-1864); archaeological excavations in the Holy City and the appropriation by the Russian Empire of the main archeological symbols of Christianity (1882-1884); and finally, the construction of the Russian Orthodox Church of St. Alexander Nevsky next to the Holy Sepulchre and the reconfiguration of the Middle Eastern Orthodoxy (1885-1896).

\section{INDEX}

Mots-clés : Empire russe, Empire ottoman, Jérusalem, Saint-Sépulcre, « Domaine copte », église Saint-Alexandre Nevsky

Keywords : Russian Empire, Ottoman Empire, Jerusalem, Holy-Sepulchre, “Domaine copte”, St. Alexander Nevsky church

\section{AUTEUR}

\section{ELENA ASTAFIEVA}

CERCEC (CNRS-EHESS)

elena.astafieva@ehess.fr 